\title{
A PROTOTYPE SYSTEM FOR ACQUISITION AND ANALYSIS OF 3D MANDIBULAR MOVEMENT
}

\begin{abstract}
This paper presents the development of a new prototype system for acquisition and analysis of the 3D mandibular movement.

In Dental Medicine, the study of the mandibular movement is very important in oral rehabilitation treatments, since it allows the diagnosis of clinical pathologies in the temporomandibular joints and therefore, it helps medical doctors to select the most adequate treatment plan and its future assessment.

In this work, a facial arc commonly used in Dental Medicine, was adapted to incorporate electromagnetic sensors to measure the movement of the mandible. Thus, some parts of the arc were redesigned, in order to ensure the best comfort for patients, and a compatible support for the sensors were designed.
\end{abstract}

A complete new user interface was also developed to display and analyse the 3D movement acquired. This software integrates a neural network algorithm to accomplish the conversion of the output voltage of the electromagnetic sensors employed into 3D cartesian coordinates.

The prototype developed reveals to be easy to use, comfortable for patients and capable of being produce at an affordable price.

\section{1 - INTRODUCTION}

The human mandible and the temporomandibular joint make an interesting and complex biomechanical system that performs several functions and produces forces of considerable value and high precision. The peculiar construction of the temporomandibular joint allows the mandible to move according to six degrees of freedom. In Dental Medicine, it is essential to know the mandibular kinematics in order to simulate the temporomandibular joint, place correctly teeth moulds in articulators, reproduce the mandibular movements and consequently, the satisfactory resolution of occlusion problems.

With so high importance in Dental Medicine, it is normal that many devices have being developed to acquire the mandibular movement. However, the existent devices are not friendly to use and have prices that are not affordable to be used in common medical dental centres.

To overcome these difficulties, we developed a new prototype system to acquire and analyse the mandibular movement that is easy to use and that can be produced with an affordable price.

The experimental results obtained using our device permits us to conclude that it can be successfully used to acquire and analyse the mandibular movement. Moreover, as it uses common dental facial arcs as its main support structure as well as it is based on technologies of low price, its final price will substantially lower than the existent devices. With these two important characteristics, we conclude that our prototype is suitable for being used in usually medical dental centres helping dental doctors in their diagnosis and treatment plans. 
This paper is organized as follows: in the next section, some of the existent devices for the acquisition of the mandibular movement are indicated; afterwards, our prototype system is described; then, in section fourth, the user interface that is used to control the acquisition process and analyse the results obtain is describe; the conversion between the output voltage of each sensor used and the associated Cartesian coordinates is addressed in the section fifth; finally, in the last section, some main conclusions are pointed out and perspectives of future work are indicated.

\section{2 - EXISTENT DEVICES}

Enciso et al., in (Enciso, 2002) and Hugger et al., in (Hugger, 1999), describe two commercial products for the acquisition of the 3D mandibular movement that use ultrasonic sensors to acquire the trajectory of the incisive point. Leader et al., in (Leader, 2002), Mesnard et al., in (Mesnard, 2003), and Kinuta et al., in (Kinuta, 2003), use optical systems to record the same movement, and Garcia et al., in (Garcia, 2003), describes a commercial system which is based on electromagnetic sensors for the same purpose.

However, both commercial and academic devices are considered very expensive and difficult to use in common clinical situations, (Santos, 2005). Having these in mind, we developed a new prototype system for the acquisition and analysis of the 3D mandibular movement that can be produced a reasonable price, with an easy modus operandi and accuracy similar to the ones offer by the existent devices.

\section{3 - OUR PROTOTYPE}

The development of our prototype system was divided in three major phases: the choice of the technology to employ, the design and production of the components for its support structure and the development of the software application control the movement acquisition and display and analysis the movement acquired.

In order to obtain a high quality standard product, in the development of the prototype system it was adopted a "structured product development process", (Ulrich, 2000). Thus, during personal interviews with medical dentists were found out the customer needs and, with a market research done in parallel, were pointed out the product specifications that should be addressed by the new system. Then, the concept and the product architecture were defined and finally, using rapid prototyping techniques, prototype components were produced, assembled and fully tested.

During the personal interviews done with the medical dentists, it was frequently referred by them that the actual equipments are very expensive, uncomfortable for patients and difficult to use. Having these disadvantages in mind, the main product specifications for our system were defined as:

1. it should acquire the 3D movement of the incisive point;

2. the user should be able to visualize and analyse the movement acquired;

3. it should be comfortable for patients and easy to use;

4. its production should assure an affordable price.

The first phase in the development of our prototype was concerned with the selection of the technology to be used in the acquisition of the mandibular movement, since the main support 
structure of the system would strongly depend on the choice considered. After a technology survey, electromagnetic sensors based on giant magneto resistive (GMR) principle reveal to be the best option for this application. GMR sensors can measure the magnetic field created by a small magnet placed inside the patients' mouth (located nearly to the incisive point). These sensors allow the measurement of the movement involved without contact, are very compact and have low price.

The selected sensors, Fig. 1, present a low current consumption, a wide supply voltage, small size (5.99 x $4.90 \times 1.55 \mathrm{~mm})$ and have an excellent sensitivity. Table 1 , summarizes the main characteristics of the model selected.

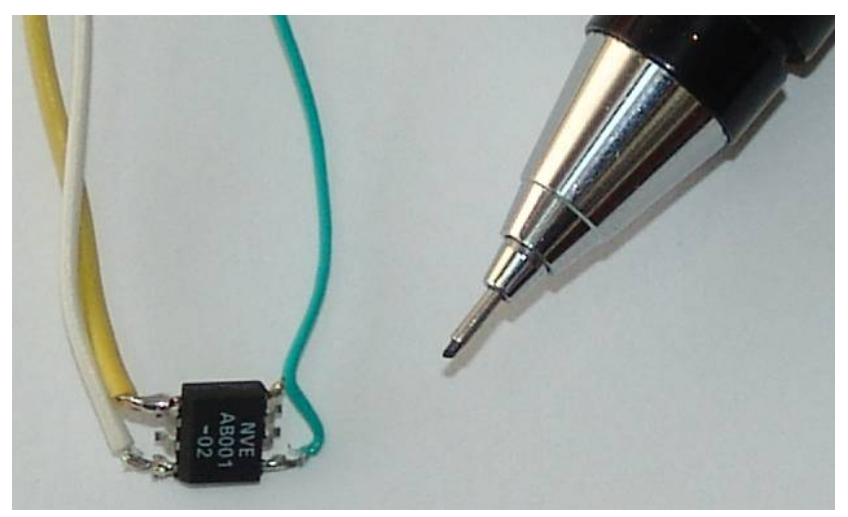

Fig. 1: Electromagnetic sensor employed in our prototype to acquire the mandibular movement.

Table 1: Main characteristics of the sensors used.

\begin{tabular}{|l|c|c|c|}
\hline \multicolumn{1}{|c|}{ Property } & Minimum & Maximum & \\
\hline Supply voltage & $<1$ & \pm 25 & Volts \\
\hline Bandwidth & DC & 1 & $\mathrm{MHz}$ \\
\hline Working temperature & -50 & 125 & ${ }^{\circ} \mathrm{C}$ \\
\hline Wheastone bridge Offset & -4 & +4 & $\mathrm{mV} / \mathrm{V}$ \\
\hline No - linearity & & 2 & $\%$ (unipolar) \\
\hline Histeresys & & 4 & $\%$ (unipolar) \\
\hline
\end{tabular}

Since the sensors choose have just a single sensitive axis, in order to acquire the 3D movement of the mandible it was necessary to use three sensors mounted perpendicularly. Thus, to assembly the sensors in their relative position two circuit boards were developed, Fig. 2. 


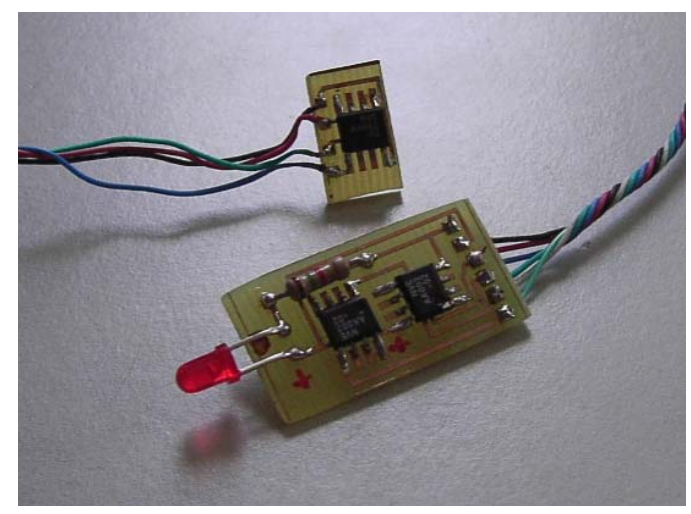

Fig. 2: Circuit boards developed to assembly the sensors.

After having chosen the technology to be used in our prototype, it was defined its support structure. In order to reduce the development time and the final cost of our product, instead of design a completely new support structure, we decided to adapt a facial arc as its main support structure.

Among the existent commercial facial arcs, it was selected a model that is recent, compatible with several common dental articulators and that is already available in many medical dental centres, Figs. 3 and 4.

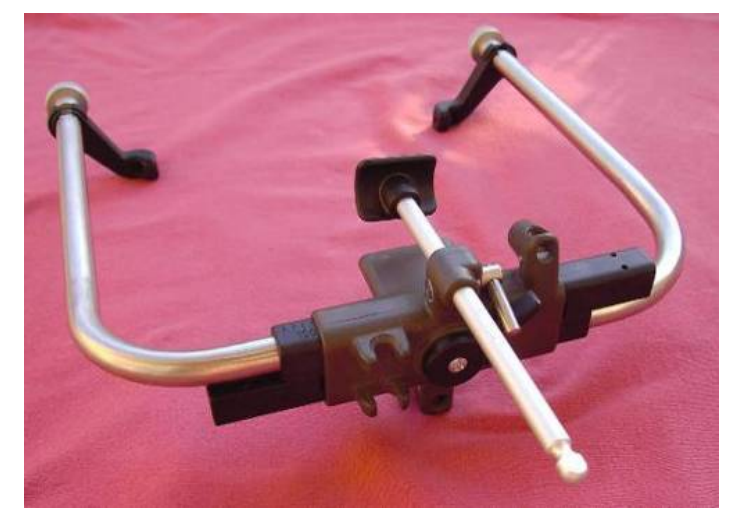

Fig. 3: Facial arc used as the main support structure of our prototype.

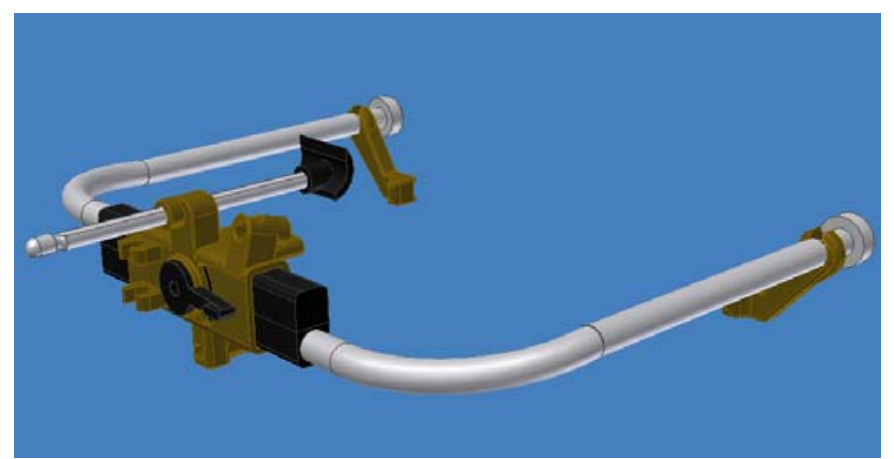

Fig. 4: 3D CAD model of the facial arc adapted as the main support structure of our prototype. 
As the selected facial arc was originally developed to be used in static measurements, its auricular parts are not particularly comfortable for the patients when they are performing the movement of their mandibular, Fig. 5. Thus, we decided to redesign the auricular parts making them more appropriate to be used in dynamic measurement procedures.

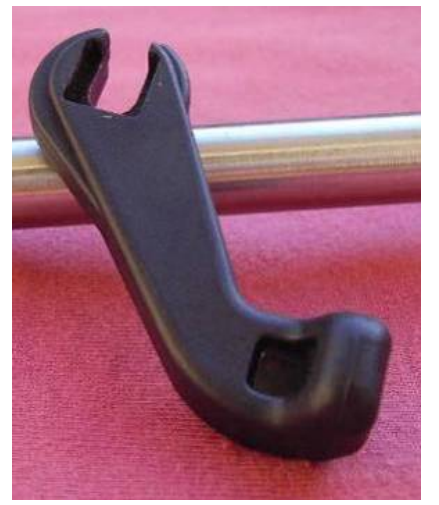

Fig. 5: Original auricular component of the facial arc.

The general dimensions and the groove of the original auricular components were maintained, but a more organic shape replaced the original one so it could offer to the patients a higher sensation of comfort; thus, spheres replaced the end parts of the original auriculars. The new auricular components, presented in Figs. 6 and 7, have also the additional advantage of being easier to clean.

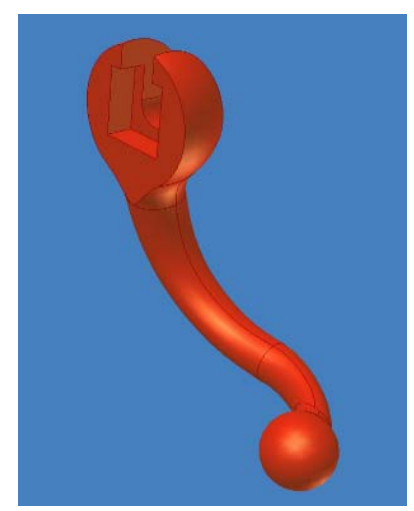

Fig. 6: Redesigned auricular component.

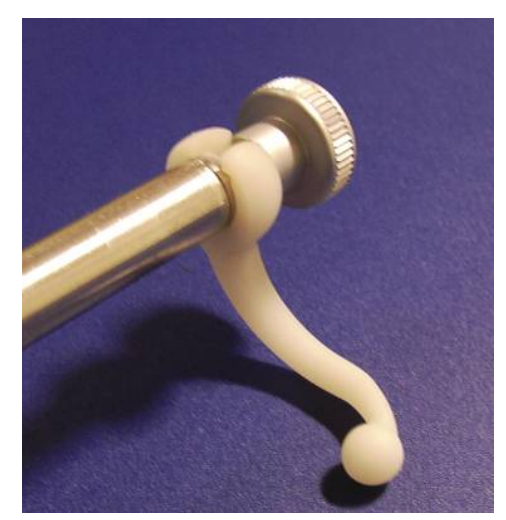


Fig. 6: Prototype of the new auricular component.

The support of the electromagnetic sensors employed in our system to acquire the mandibular movement was designed to meet the following main requirements: be adaptable to the facial arc adopted, be able to accommodate the two circuit boards developed for the magnetic sensors, and to be lightweight. To satisfy these needs, the most appropriate shape was also an organic one, Fig. 8.

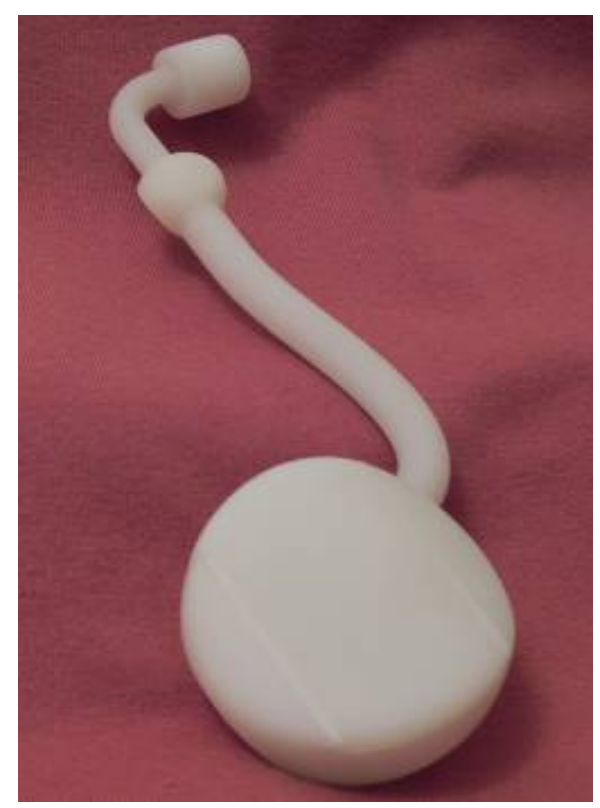

Fig. 8: Support designed for the sensors.

To fix the sensors support to the facial arc it was designed a specific notch that fits in the groove already existing in the facial arc. This notch also prevents the translation and rotation of the sensors support, Fig. 9.

It is important to refer that it is possible to redesign the sensors support so it can be adaptable to other commercial facial arcs as well.

The end part of the sensors support was enlarged to accommodate the data acquisition cable, Fig. 9. This area of the sensors support can be used in the future to accommodate a wireless transmitter. 


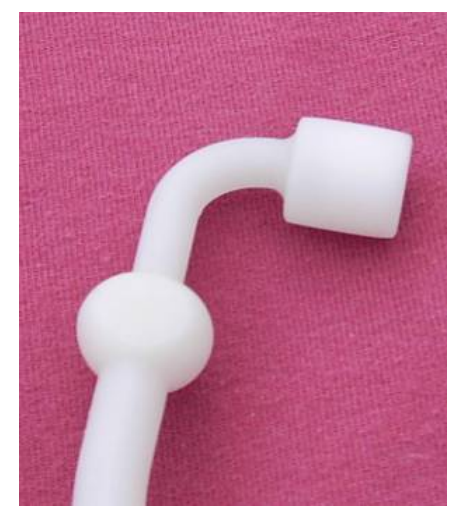

Fig. 9: Detail of the sensors support.

The integrated circuit boards, Fig. 2, are assembled perpendicularly inside the sensors support house by pressure. The sensors house is then closed by a sliding cover. This cover presents a hole in its top to accommodate a led that is used to indicate if the acquisition system is on.

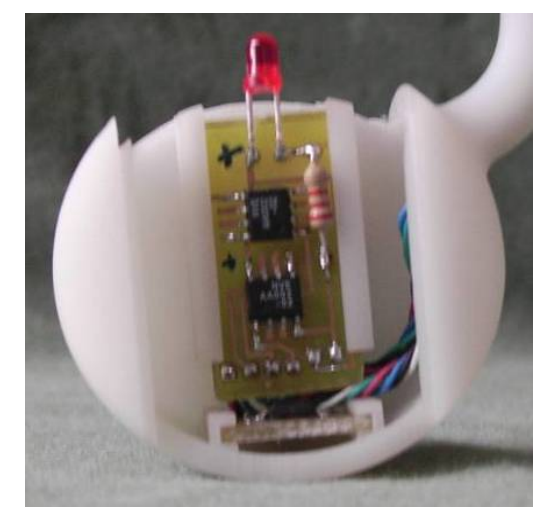

Fig. 10: Inside view of the sensors support house.

For the sensors signals acquisition, it was used a data acquisition device (DAQ) with plugand-play USB connectivity. The selected DAQ is light, small, low priced and easy to use. It has four differential analog channels (or eight single ended), 12 bit resolution and acquisition rate up to $10 \mathrm{kS} / \mathrm{s}$. In this work, to reduce the electrical noise, the sensors were connected using the differential mode.

In spite the selected $D A Q$ could be used to directly power the electromagnetic sensors, it was used an external power supply from EMS instead. The main reason of this decision was because with the external power supply the output signals of the sensors used could be increased and so the noise effects reduced.

Fig. 11 shows the interior of the interface box, which contains the DAQ and the power supply referred. 


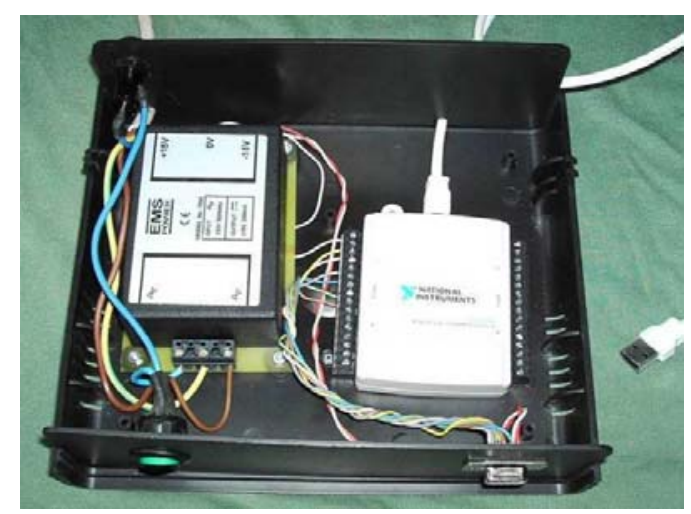

Fig. 11: Interior of the interface box.

\section{4 - USER INTERFACE}

To make easy and friendly the procedures to accomplish the acquisition and analysis of the 3D mandibular movement using our prototype, it was built a new software application with an adequate interface, Figs. 12 and 13. In the upper part of the interface designed, the user can write the patients' personal data: name, age, sex and missing teeth. The buttons presented in the interface permit the full control of the acquisition process, as well as, the generation of reports of the medical exams performed that can be printed or saved, Fig. 14.

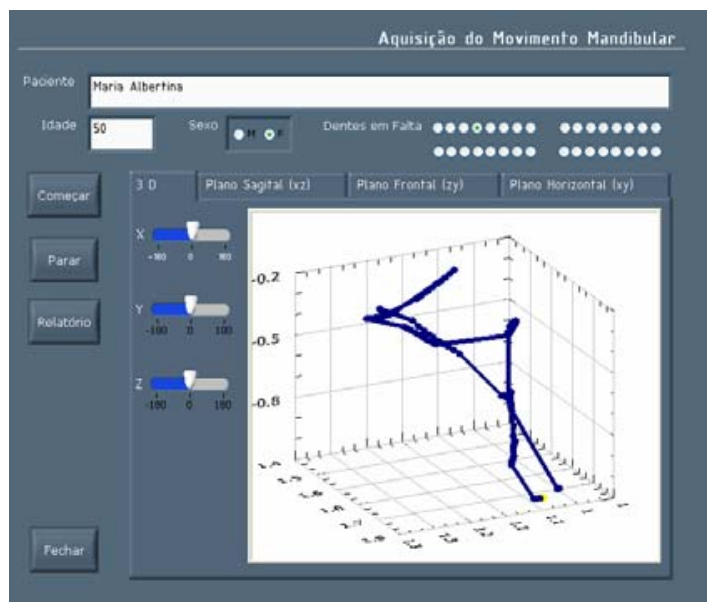

Fig. 12: User interface of our prototype device: 3D graphic.

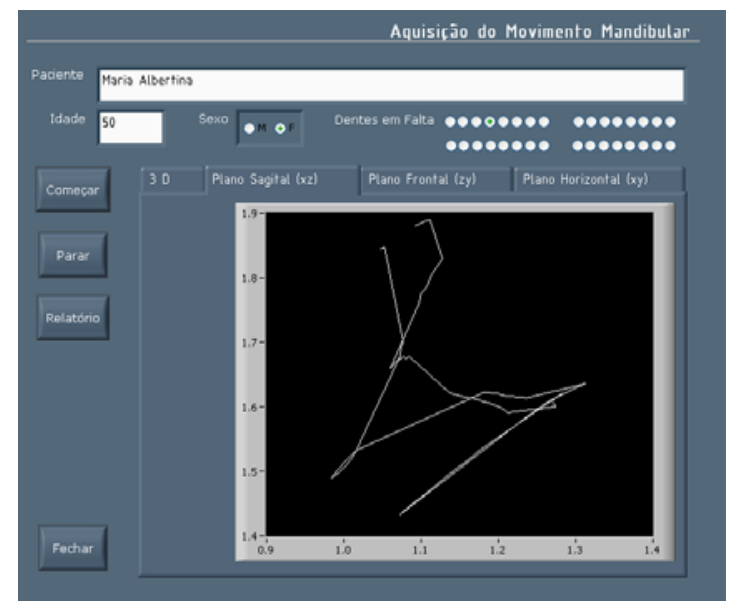


Fig. 13: User interface of our prototype device: 2D graphic in the sagital plane.

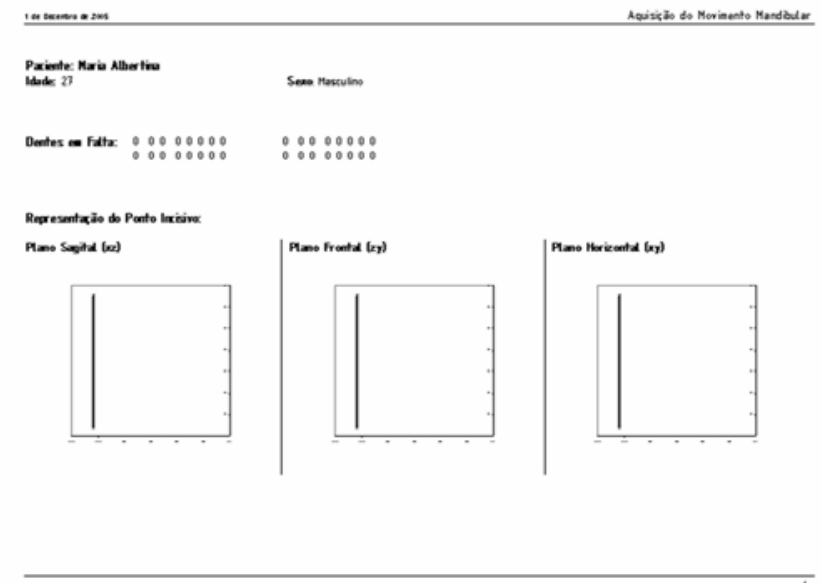

Fig. 14: User interface of our prototype device: template for the exam reports.

The results can be visualized according with four graphs commonly used in Dental Medicine: one with the 3D acquired trajectory of the incisive point (Fig. 12), and three 2D graphs with the associated projections in the sagital (Fig. 13), frontal and horizontal planes. Moreover, the user can easily rotate and zoom the 3D graph displayed.

To obtain the 3D trajectory of the magnet placed inside the patients' mouth, it is necessary to convert the output voltage of the sensors in 3D cartesian coordinates. Also, while testing the system developed, it was verified that when the magnet is moved along one direction, the output voltages of all three sensors vary; that is, to each position in space (coordinates $\mathrm{x}, \mathrm{y}$ and z) corresponds a set of three voltages. To solve this conversion problem, a neural network was employed.

\section{5 - CONVERSION VOLTAGES/COORDINATES}

Neural networks are inspired in the biological nervous systems. Thus, they are composed of simple elements operating in parallel, and can be trained to perform a particular function by adjusting the values of the connections (weights) between these elements, (Demuth, 2005) and (Marques, 1999).

In this work, a radial basis neural network was used because this type of network can be trained more quickly and it is possible to interpret the contribution of each unit in the global behavior of the associated net, as each layer is only active in a limited zone of the entry space, (Marques, 1999).

The neural network implemented is composed by two layers: a hidden radial basis layer of $S^{1}$ neurons, and an output linear layer of $S^{2}$ neurons, Fig. 15. The transfer functions adopted in our neural network are plotted in Fig. 16. 


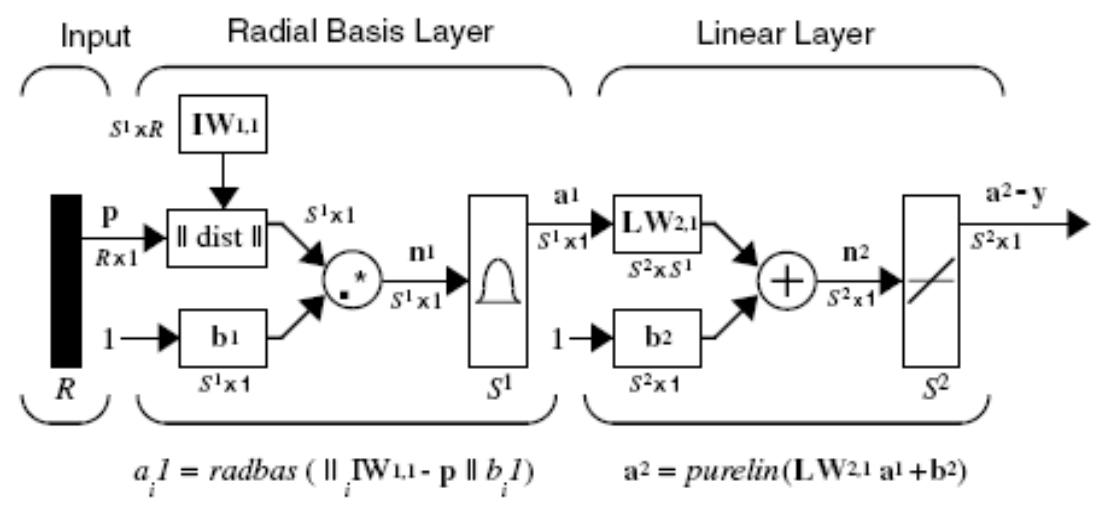

Fig. 15: Neural network used in our prototype to the conversion voltages/coordinates (adapted from (Demuth, 2005)).
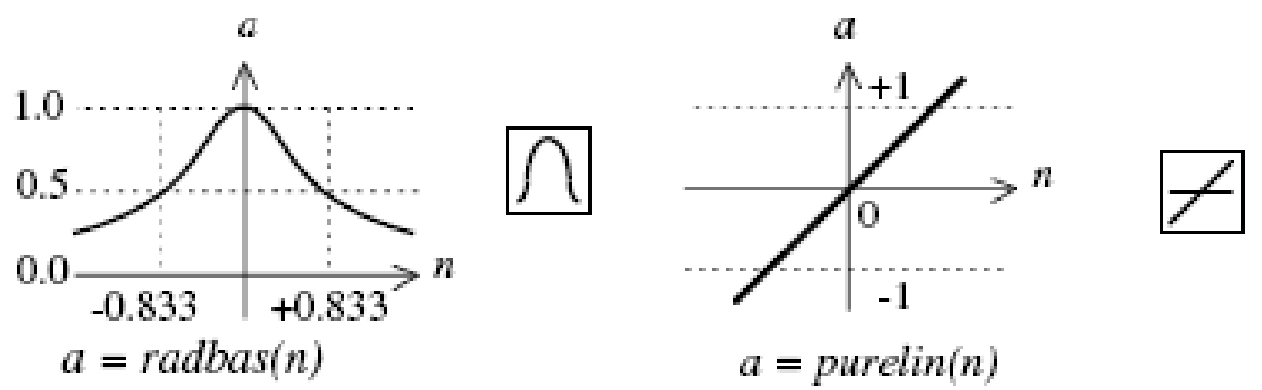

Fig. 16: The transfer functions used in the neuronal network used in our prototype (adapted from (Demuth, 2005)).

The data used in the training of the neuronal network used in our prototype device was acquired by measuring the output voltages on calibration points that were defined regularly spaced within a 3D volume. The step size adopted along each axis for the calibration points was $5 \mathrm{~mm}$ and the volume dimensions were defined as $70 \times 40 \times 40 \mathrm{~mm}$, which is according with the normal limits of the mandibular movement. The measured voltages and the associated 3D catesian coordinates were then used as "inputs" and "targets" in the training phase of the radial neural network developed.

The neural network built and trained was then integrated into the user interface application of our prototype device.

Fig. 17 shows our complete system for the acquisition and analysis of the 3D mandibular movement. Our system is easy to use and very comfortable for patients, Fig. 18. 


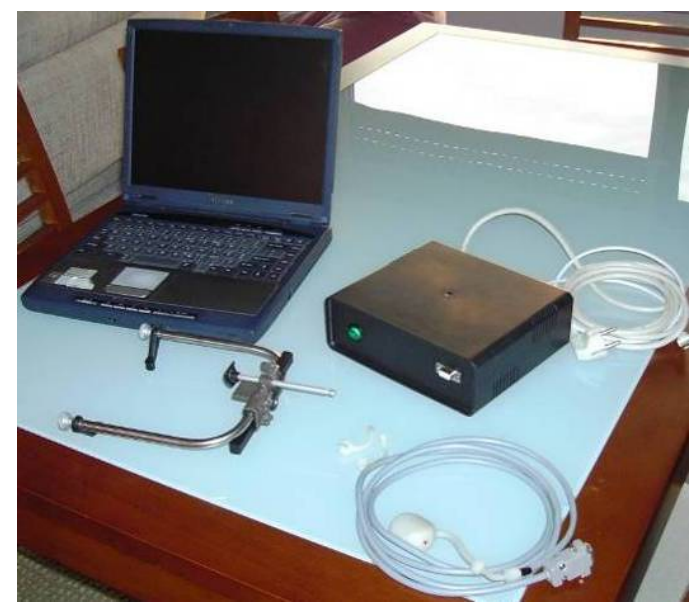

Fig. 17: Prototype system developed for acquisition and analysis of the 3D mandibular movement.

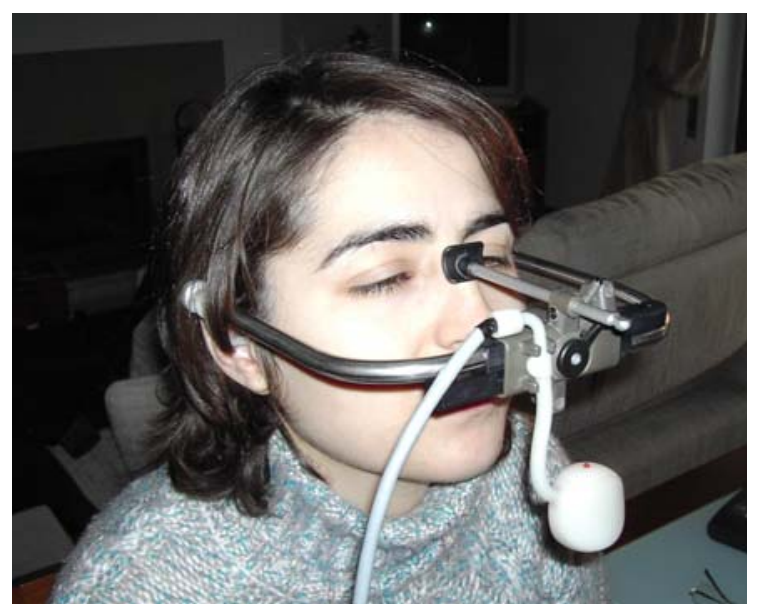

Fig. 17: The usage of the system developed.

\section{6 - CONCLUSIONS AND FUTURE WORK}

It was successfully developed a low cost prototype system for acquisition and analysis of the 3D mandibular movement that has an easy modus operandi and is comfortable for the patients and doctors. This system can be a useful tool to help dental doctors to diagnosis and define adequate treatment plans for problems involving dental occlusions.

The GMR sensors used in our prototype to acquire the incisive point trajectory have very low price, small size and do not require contact. These properties make them an ideal solution for this king of application, requiring only that a small magnet is placed inside the patients' mouth.

A facial arc commonly used in Dental Medicine was adapted as the main support structure of our acquisition prototype. Some parts of this arc were then redesigned so they could be more comfortable for the patients in this new dynamic use. This adaptation is responsible to low considerable the final price of our prototype.

To accommodate the three magnetic sensors used was designed a specific support that can be easily fixed in the facial arc. 
To control the acquisition process, display and analyze the mandibular movement acquired it was developed a completely user interface application that has a friendly interface. This application runs on personal computers and permits the store of the patients' personal data and the generation of medical exam reports as well.

To convert the output voltage of the sensors used to the associated 3D coordinates, a radial neural network was implemented and integrated in the user interface application.

As future work we can indicate the employment, test and comparison of more complex, robust and precise neural network to accomplish the conversion of the output voltage of the sensors to the associated 3D Cartesian coordinates, the use of acquisition boards with higher resolution and precision and the consideration of electromagnetic sensors that have more than one axis of sensitivity. This comparison is very important as the resolution and precision of our prototype system is highly depending on the quality of the elements used in the movement acquisition and pos-processing.

The deeply use of our prototype in real clinical environments is also an important task that should be addresses in the near future and that should be strong emphasis.

\section{REFERENCES}

(Demuth, 2005) Demuth H., Beale M., Hagan M., "Neural network toolbox for use with MATLAB”, The Mathworks, 2005.

(Enciso, 2002) Enciso R., Menon A., Fidaleo D., Neumann U., Mah J., "The virtual craniofacial patient 3D jaw modeling an animation", Studies in Health Technology Informatics, vol. 94, pp. 65-71, 2002.

(Garcia, 2003) Garcia R., Oliveira V., Cury A., "Short term evaluation of interocclusal distance during speech after new removable prosthesis insertion”, Journal of Applied Oral Science, vol. 11, no. 3, pp. 216-222, 2003.

(Hugger, 1999) Hugger A., Bölöni E., Berntien U., Stüttgen U., “Accuracy of an ultrasonic measurement system for jaw movement recording”, 35th Annual Meeting of the Continental European Division of the International Association for Dental Research, Montpellier, France, 1999.

(Kinuta, 2003) Kinuta, S., Wakabayashi, K., Sohmura, T., Kojima, T., Nagao, M., Nakamura, T., Takahashi, J., "Simple system to record jaw movements by a home digital camcorder", The International Journal of Prosthodontics, vol. 16, no. 5, pp. 563-569, 2003.

(Leader, 2002) Leader, J., Boston, J., Debski, R., Rudy T., "Mandibular kinematics represented by a non-orthogonal floating axis joint coordinate system”, Journal of Biomechanics, vol. 36, no. 2, pp. 275-281, 2002.

(Marques, 1999) Marques J., “Reconhecimento de padrões métodos estatísticos e neuronais”, IST Press, 1999.

(Mesnard, 2003) Mesnard, M., Ballu, A., Cid, M., "Méthode et validation pour l'articulation temporo-mandibulaire”, 16ème Congrès Français de Mécanique, Nice, France, 2003.

(Santos, 2005) Santos, I., "Desenvolvimento de um sistema protótipo para a aquisição e análise do movimento mandibular”, MSc Thesis in Industrial Design by University of Porto, Portugal, 2005. 
(Ulrich, 2000) Ulrich K., “Product design and development”, McGraw Hill, 2000. 